\title{
Lunar Surface Roughness Estimation Using Stereoscopic Data
}

\author{
By Tomoyuki YOSHIMATSU, ${ }^{1)}$ Akira IWASAKI, ${ }^{1)}$ Junichi HARUYAMA, ${ }^{2)}$ Makiko OHTAKE, ${ }^{2)}$ Tsuneo MATSUNAGA ${ }^{3)}$
}

\author{
1) Department of Aeronautics and Astronautics, The University of Tokyo, Tokyo, Japan \\ 2) Japan Aerospace Exploration Agency, Institute of Space Astronautical Science, Sagamihara, Japan \\ ${ }^{3)}$ National Institute for Environmental Studies, Tsukuba, Japan
}

(Received April 30th, 2008)

On September 14, 2007, Selenological and Engineering Explorer (SELENE), which is a Japanese lunar polar orbiter, was launched. SELENE carries the optical instrument, which is referred to as the Lunar Imager / SpectroMeter (LISM). The LISM is composed of three sensors. One of sensors of the LISM is the Terrain Camera (TC). TC is used to produce Digital Terrain Model (DTM) from stereo observation of lunar surface. In this work, the lunar surface bidirectional reflectance is investigated from images acquired by the TC. The Radiance Ratio (RR) of surface-reflected solar radiance measured from two view angles is obtained. The estimation of lunar surface roughness using images of the TC is discussed. As a result, it is found that the moon surface is not based on Lambert's law.

Key Words : SELENE, Lunar Surface Roughness, Radiance Ratio (RR), Digital Terrain Model (DTM)

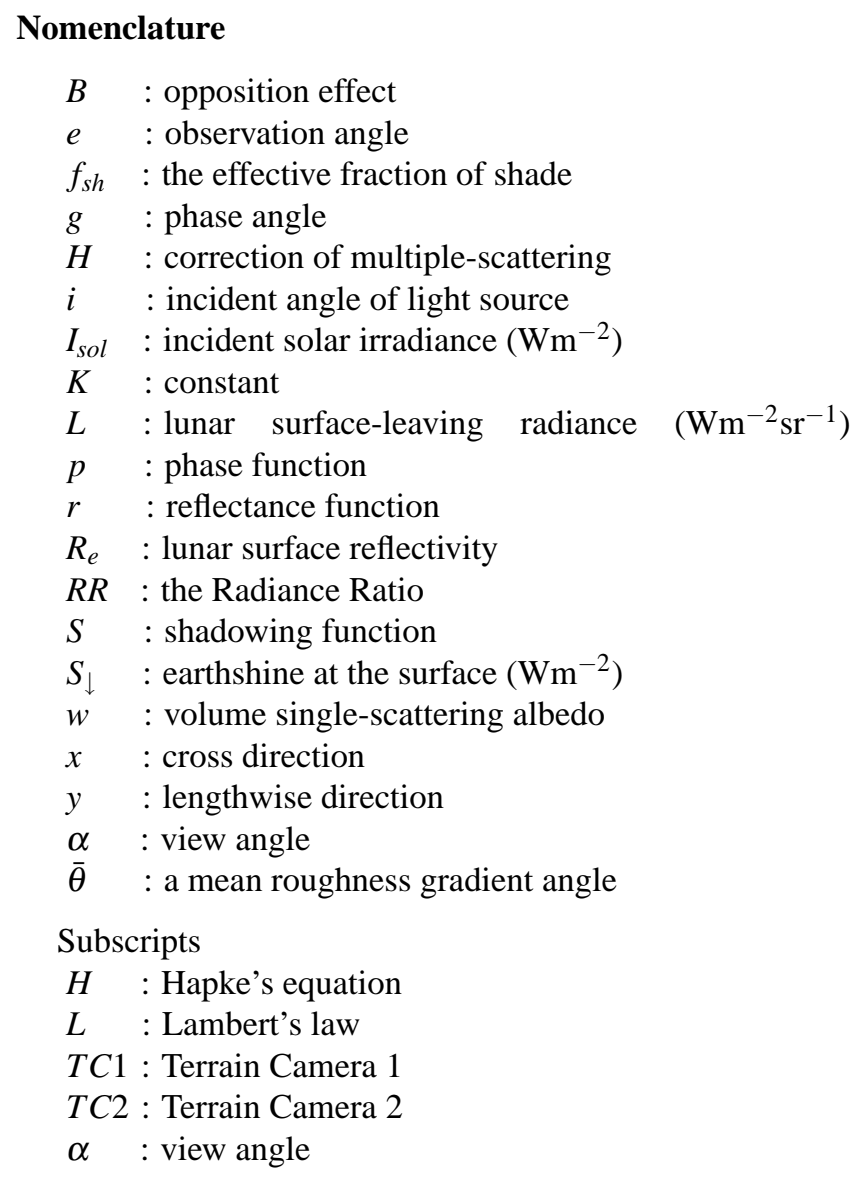

\section{Introduction}

Selenological and Engineering Explorer (SELENE), which is a Japanese lunar polar orbiter, was launched on September 14, 2007. SELENE project is a largest lunar exploration project since Apollo program from 1961 to 1975 by The National Aeronautics and Space Administration (NASA). The purpose of SELENE is to acquire lunar scientific data and to investigate the possibility of future unmanned and manned activities on the Moon. ${ }^{1,2}$ A nominal mission altitude is $100 \mathrm{~km}$, and its mission will last at least 1 year. Of the 14 mission instruments installed on SELENE, 2,3) three optical instruments share structures and electric circuits to reduce mission resources such as mass and volume: the Terrain Camera (TC), the Multiband Imager (MI) and the Spectral Profiler (SP) .1) This group of optical instruments is generally referred to as the Lunar Imager/SpectroMeter (LISM). The TC is a push-broom imager with $10 \mathrm{~m}$ spatial resolution and two slant optical heads (slant angles of $\pm 15^{\circ}$ ), which obtains two stereoscopic images in visible wavelength (ref. Fig. 1).1,4-9)

One of the most significant achievements of the SELENE mission will be global $10 \mathrm{~m}$ resolution relief maps from the TC data since the maximum area covered by such highresolution maps is currently only $20 \%$ or less. ${ }^{10)}$ Furthermore, remotely sensed stereoscopic optical data are used to obtain bidirectional reflectance. In this work, images of TC obtained at the different view angles are investigated. The registration of two images is a key issue of the comparison. Application to the lunar surface roughness estimated from images acquired by the TC is addressed using the method, which estimates bidirectional properties using the Radiance Ratio (RR) of surface-reflected solar radiance measured from two view angles.

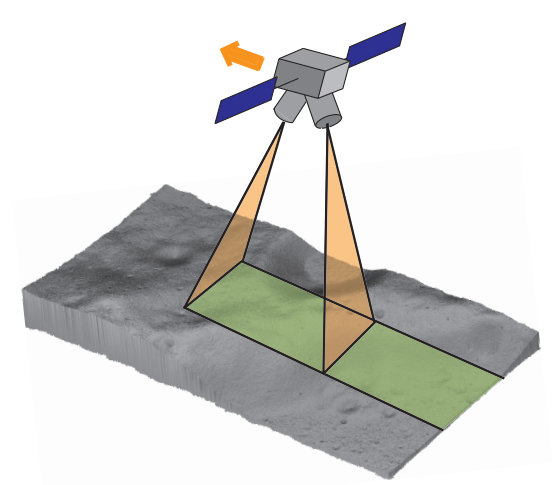

Fig. 1. Image acquisition by the TC of SELENE. 
Table. 1. TC specifications. ${ }^{11}$

\begin{tabular}{|c|c|}
\hline & $\mathrm{TC}$ \\
\hline & TC1 and TC2 \\
\hline Focal length & $72.5 \mathrm{~mm}$ \\
\hline F number & 4 \\
\hline Field of view & $19.3^{\circ}$ \\
\hline Spatial resolution/pixel & $10 \mathrm{~m}$ \\
\hline Swath width (mode) on ground & $35 \mathrm{~km}$ (nominal swath mode) \\
\hline & 40 km (full swath mode) \\
\hline & $17.5 \mathrm{~km}$ (half swath mode) \\
\hline Optics & Two refractive optical heads \\
\hline Off-nadir angle & $+/-15^{\circ}$ (slant angle of optical heads) \\
\hline Detector & 1D CCD (4096 pixels) \\
\hline Pixel size & $7 \times 7 \mu m$ \\
\hline Number of bands & 1 \\
\hline Band assignment & $430-850 \mathrm{~nm}$ \\
\hline Quantization & 10 bit \\
\hline MTF at Nyquist freq. & $>0.2$ \\
\hline Integration time (mode) & $1.625 \mathrm{~ms}$ (Short exposure mode) \\
\hline & 3.25 (Middle exposure mode) \\
\hline & 6.5 (Long exposure mode) \\
\hline Data compression mode & $\begin{array}{l}\text { Compression mode using } \\
\text { discrete cosine transform method } \\
\text { (lossy) and non-compression mode }\end{array}$ \\
\hline Q table for nominal observation & 18 patterns \\
\hline $\mathrm{H}$ table for nominal observation & 1 pattern \\
\hline
\end{tabular}

\section{Lunar Surface Reflectance}

\subsection{Lambert, hapke and roughness}

One of reflectance functions widely used is known as Lambert's law. Lambert's law is based on the empirical observation that the brightness of surface is nearly independent of $e$ and $g$, which means that the brightness of any surface must be proportional to $\cos i$. Lambert's law is described as

$$
r_{L}(i, e, g)=K_{L} \cos i
$$

Although no natural surface obeys Lambert's law exactly, many surfaces approximate the scattering behavior described by this law. The equation described by Hapke is one of the famous reflectance functions and used widely. Hapke's equation is based on the single scattering by particulate medium and the empirical observation, which have many parameters called Hapke parameters. Hapke's equation is described as

$$
\begin{aligned}
r_{H}(i, e, g)= & \frac{w}{4 \pi} \frac{\cos i}{\cos i+\cos e}\{[1+B(g)] p(g) \\
& +H(\cos i) H(\cos e)-1\} S(i, e, g, \bar{\theta}) .
\end{aligned}
$$

The function $S$ represents the effects of gradient of plane from resolution of sensor to particle size, corresponding to surface roughness.

\subsection{Influence of roughness}

The Radiance Ratio (RR) method is more appropriate for lunar surface roughness than earth surface roughness because there is no atmosphere around the moon and the lunar surface reflectivity is approximately constant because the lunar surface is covered by regolith.
Assuming that lunar surface can be regarded as Lambertian reflectors, lunar surface-leaving radiance at a given view angle is described as ${ }^{12)}$

$$
L_{\alpha}=\frac{1}{\pi}\left(I_{\text {sol }}+S_{\downarrow}\right) R_{e}\left(1-f_{s h}(\alpha)\right) .
$$

Following Adams et al., ${ }^{13)}$ shade is defined here as the darkening of the surface due to both the presence of unresolved shadows and increased incidence angle of the solar irradiance. Shade thus defined varies with view angle, even for a Lambertian surface, because the visibility of shadows depends on view angle. $f_{s h}(\alpha)$ is thus inherently dependent on the sun-lunar surface-sensor geometry. For example, at constant illumination conditions, shadows seen at nadir viewing will not be visible at a down-sun viewing angle, thus making the surface appear lighter in the down-sun angle. Such lightening is expected to be proportional to $f_{s h}(\alpha=$ nadir $)$ and surface roughness, and will not occur for perfectly smooth surfaces $\left(f_{s h}=0\right)$. Furthermore, the Radiance Ratio $\left(\mathrm{RR}_{\alpha_{2}}^{\alpha_{1}}\right)$ of two lunar surface-reflected radiances measured from two view angles $\alpha_{1}$ and $\alpha_{2}$ is described as

$$
\operatorname{RR}_{\alpha_{2}}^{\alpha_{1}}=\frac{L_{\alpha_{1}}}{L_{\alpha_{2}}}=\frac{\frac{1}{\pi}\left(I_{s o l}+S_{\downarrow}\right) R_{e}\left(1-f_{s h}\left(\alpha_{1}\right)\right)}{\frac{1}{\pi}\left(I_{s o l}+S_{\downarrow}\right) R_{e}\left(1-f_{s h}\left(\alpha_{2}\right)\right)} .
$$

Assuming that $R_{e}$ does not change with $\alpha$ (Lambertian reflection), and canceling out variables that are independent of $\alpha,\left(I_{s o l}, S_{\downarrow}\right.$, and $\left.R_{e}\right)$ are canceled and Eq. (4) is re-written as

$$
\operatorname{RR}_{\alpha_{2}}^{\alpha_{1}}=\frac{\left(1-f_{s h}\left(\alpha_{1}\right)\right)}{\left(1-f_{s h}\left(\alpha_{2}\right)\right)}=\frac{L_{T C 2}}{L_{T C 1}} .
$$

Here, perfectly smooth surfaces are expected to display $\mathrm{RR}_{\alpha_{2}}^{\alpha_{1}}=1$ ( $f_{s h}=0$ at all angles), and increasingly rough surfaces are expected to display $\mathrm{RR}_{\alpha_{2}}^{\alpha_{1}}$ values diverging from unity, regardless of surface reflectivity (i.e., surface composition). Thus, the lunar surface roughness is represented by $\mathrm{RR}_{\alpha_{2}}^{\alpha_{1}}$ in Eq. (5).

\subsection{Radiance ratio method for hapke's equation}

Hapke's equation represents more accurate reflectance of planet than Lambert's law. Using Hapke's equation for RR method, the result is not simple as Eq. (5). In this case, we can obtain the following equation.

$$
\begin{aligned}
\mathrm{RR}_{\alpha_{2}}^{\alpha_{1}}= & \frac{r_{H}\left(i_{1}, e_{1}, g_{1}\right)\left(I_{\text {sol }}+S_{\downarrow}\right)}{r_{H}\left(i_{2}, e_{2}, g_{2}\right)\left(I_{\text {sol }}+S_{\downarrow}\right)} \\
= & \frac{\frac{w}{4 \pi} \frac{\cos i_{1}}{\cos i_{1}+\cos e_{1}}\left\{\left[1+B\left(g_{1}\right)\right] p\left(g_{1}\right)\right.}{\frac{w}{4 \pi} \frac{\cos i_{2}}{\cos i_{2}+\cos e_{2}}\left\{\left[1+B\left(g_{2}\right)\right] p\left(g_{2}\right)\right.} \\
& \quad \frac{\left.+H\left(\cos i_{1}\right) H\left(\cos e_{1}\right)-1\right\} S\left(i_{1}, e_{1}, g_{1}, \bar{\theta}\right)}{\left.+H\left(\cos i_{2}\right) H\left(\cos e_{2}\right)-1\right\} S\left(i_{2}, e_{2}, g_{2}, \bar{\theta}\right)} \\
= & \frac{\frac{\cos i_{1}}{\cos i_{1}+\cos e_{1}}\left\{\left[1+B\left(g_{1}\right)\right] p\left(g_{1}\right)\right.}{\cos i_{2}}\left\{\left[1+B\left(g_{2}\right)\right] p\left(g_{2}\right)\right. \\
& \quad \frac{\left.+H\left(\cos i_{1}\right) H\left(\cos e_{1}\right)-1\right\} S\left(i_{1}, e_{1}, g_{1}, \bar{\theta}\right)}{\left.+H\left(\cos i_{2}\right) H\left(\cos e_{2}\right)-1\right\} S\left(i_{2}, e_{2}, g_{2}, \bar{\theta}\right)}
\end{aligned}
$$


According to Eq. (6), RR method using Hapke's equation is affected by many phenomena, such as suface gradient, opposition effect, multiple scatter. Thus, RR method using Lamber's law is not accurate, and we expects that the results from that method is not so good. One of the purpose of this paper is to confirm this expectation, and indicates that the lunar surface is not based on Lambert's law.

\section{Image Ortho and the Digital Terrain Model (DTM)}

The TC observes the lunar surface during the day-time of the Moon using two cameras that respectively face the slightly diagonal fore looking and aft looking of the satellite. It can take stereo images of the globe with a high resolution of $10 \mathrm{~m}$ in a push-broom mode. A panchromatic filter is attached to the optics to limit the wavelength from 430 to 850 $\mathrm{nm}$. Table 1 shows the specifications of TC). During the SELENE nominal mission of 1 year or 11 Moon cycles (about 11 times 27.5 days), TC will be operated in five Moon cycles.

Two images acquired by TC must be orthorectified to estimate the RR. Therefore, corresponding points of two images need to be matched accurately. Now, the parameter of an executable module is changed to produce ortho images and DTM. Specifically, "location of intersection point of the bundle method" is changed; the case 0 method represents the tie point on the base vector shown in Fig. 2 (a), whereas the case 1 method represents the tie point on the midpoint between two vectors shown in Fig. 2 (b). In the case 0 method, the corresponding point of $\mathrm{TC} 1$ and $\mathrm{TC} 2$ are projected to slightly different place.

When one of stereoscopic images is obtained by a nadir looking sensor, the case 0 method is appropriate because the accurate determination of line of sight is obtained. In the case of TC, both TC1 and TC2 have a tilt relative to nadir. Thus the case 1 method is more appropriate than the case 0 method because corresponding points of two images are placed midpoint between two line of sight vectors.

\section{Results and Discussion}

The images used for this study are acquired by TC at Dyson near latitudes of 61 degrees and longitude of 240 degrees on November 2, 2007. Two images of TC1 and TC2 are shown in Figs. 3 (a) and (b), which are orthorectified by the case 1 method.

\subsection{Registration of stereo image}

TC1 and TC2 images are orthorectified by the case 0 and case 1 methods because the registration of two images is important in this study. Image matching is a method to measure the relative shift of two images by calculating a cross correlation coefficient. TC2 image is assumed as a master image and TC1 image is a slave. The slave image is moved around the master image and cross correlation coefficients are calculated iteratively between TC1 and TC2 image. Two images are matched at location having maximum correlation coefficient for both orthorectifying methods. After image macthing, correlation coefficients in the $x$ and $y$ directions are calculated with a window size of $11 \times 11$ pixels and the registration errors for each direction are estimated, as shown in Fig. 4.

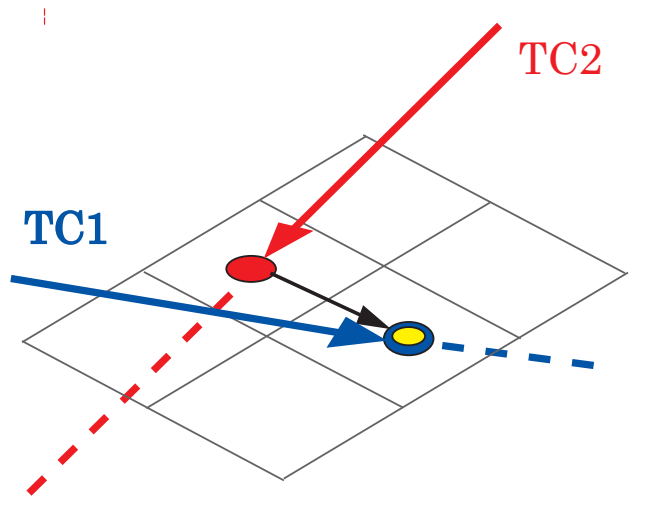

(a) the case 0 method.

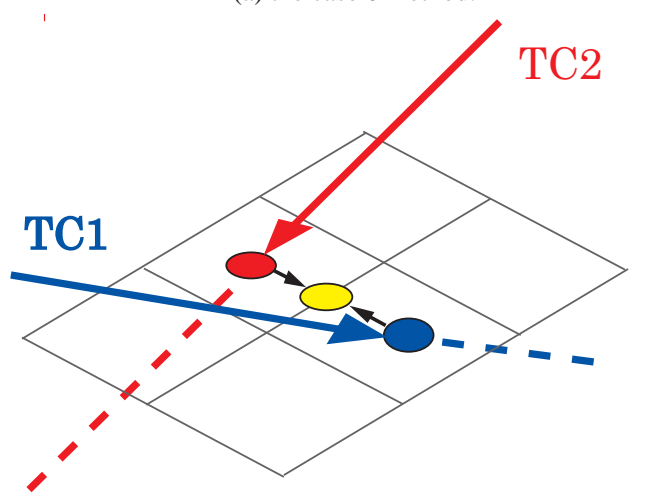

(b) the case 1 method.

Fig. 2. Tie point of TC1 and TC2.

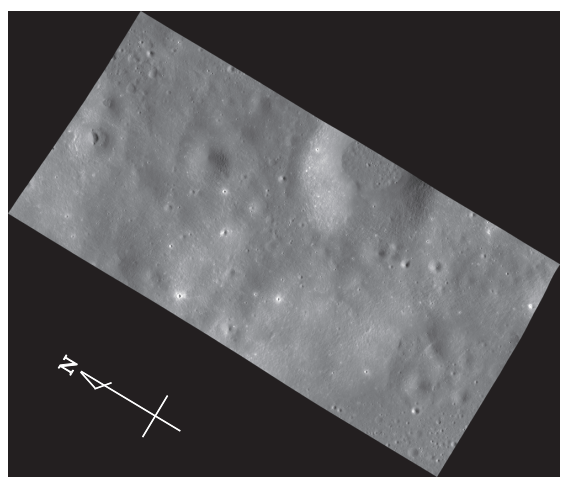

(a) TC1 image.

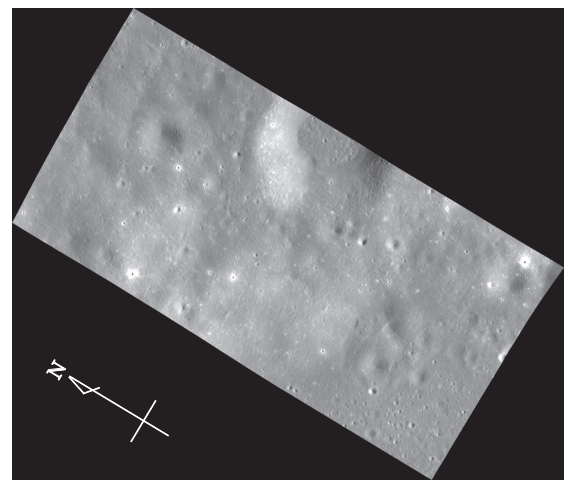

(b) TC2 image.

Fig. 3. Images orthorectified at Dyson (the case 1 method). 

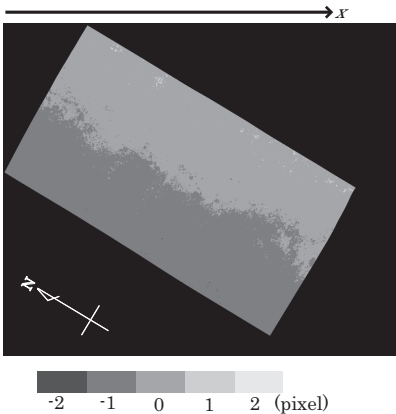

(a) image of the case 0 method. ( $x$ direction)
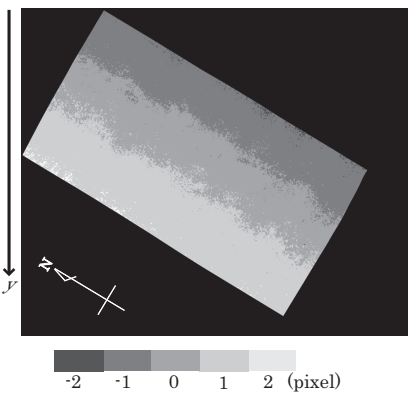

(c) image of the case 0 method. ( $y$ direction)

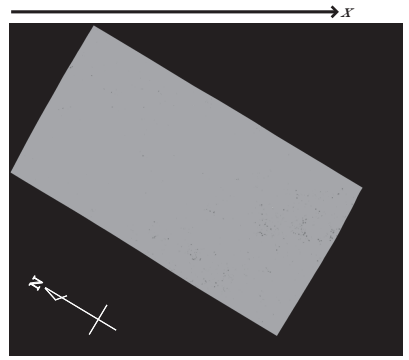

$\begin{array}{lllll}-2 & -1 & 0 & 1 & 2\end{array}$ (pixel)

(b) image of the case 1 method. ( $x$ direction)
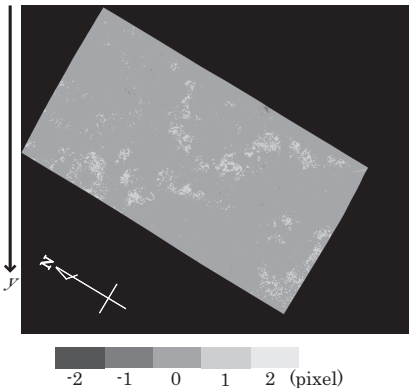

(d) image of the case 1 method. ( $y$ direction)
Fig. 4. Registration errors in $x$ direction and $y$ directions estimated by the case 0 and the case 1 methods.

It is found that the case 0 method has the registration errors of \pm 1 pixel to both directions (see Figs. 4 (a) and (c)). In contrast, it is found that the result of the case 1 method has almost no registration errors in both directions (see Figs. 4 (b) and (d)). Therefore, it is indicated that the midpoint between two vectors should be used in the orthorectification of TC. Figure 5 shows registration error distribution, which represents combining the registration errors in $x$ and $y$ directions in the case 0 method. It indicates that there are only registration errors in the cross track direction. For this reason, sight vectors of two images should be corrected in the cross track direction when the case 0 method is used.

\subsection{DTM and radiance ratio}

Tie point of TC1 and TC2 should be placed on the midpoint of two vectors for orthorectifying images of TC. Figure 6 shows the RR image produced from orthorectified images. The border line can be seen in the center of the image. This borderline issue is due to gain and offset parameters during initial checkout period. Since LISM/TC is a sensor for DTM processing and works in the JPEG compression mode in most time, the radiometric accuracy is not required. Our analysis is the special case to use the radiometric performance that should be guaranteed after the radiance ratioing. Fig. 7 shows DTM produced from TC1 and TC2 images. On the large crater indicated by red circle in Figs. 6 and 7, the

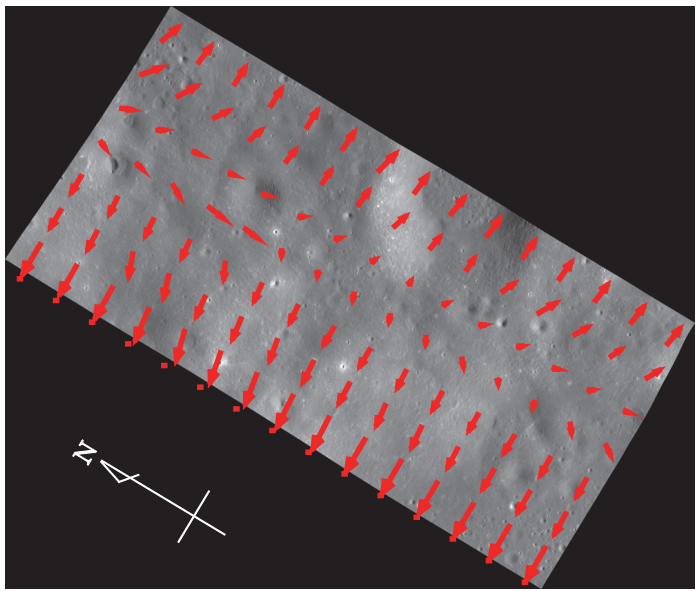

Fig. 5. Registration error distribution.

$\mathrm{RR}$ is small at the north wall inclined toward the south direction and is large at the south wall inclined toward the north direction. This means that the RR is also much influenced by observation angle because good correlation is observed in Figs. 6 and 7. The RR is not constant and depends on observation angle and roughness of surface. This shows that there exists deviation from Lambertian reflectance model. However, further discussion is needed to distinguish roughness effect from other effect, such as observation angle.

Therefore, we investigated the relationship between the $\mathrm{RR}$ and gradient derived from DTM. The mean RR for each slope angle $(d x, d y), \overline{R R}(d x, d y)$ is discribed as,

$$
\overline{R R}(d x, d y)=\frac{1}{n} \sum_{i} R R_{i}(d x, d y) .
$$

Here, $n$ is the total number that RR are added. Figure 8 shows the mean RR for each slope angle. Dark area at $\mathrm{P}$ surrounded by blue square is observed in Fig. 8. This area corresponds to blue points observed at dark area in Fig. 9. Meanwhile, bright area at $\mathrm{Q}$ surrounded by yellow square in Fig. 8 corresponds to yellow points in Fig. 9. Many yellow points is observed at south wall of craters.

The mean RR shows the representative value, where influence of roughness is averaged. For this reason, the mean RR value for slope angle in Fig. 8 is used as a look-up table and the typical value corresponding to the slope angle obtained by DTM is plotted in Fig. 10. Fig.11 shows the combined RR image, where RR image is colored red and the mean RR image is colored green. Comparison of Figs. 6 and 10 corresponds to the deviation from the mean RR, which may be attributable to partly roughness effect. Since large deviations represented by deep green or red points are found around the craters in Fig. 11, it is supposed that these regions are rougher because light are scattered at the surface. 

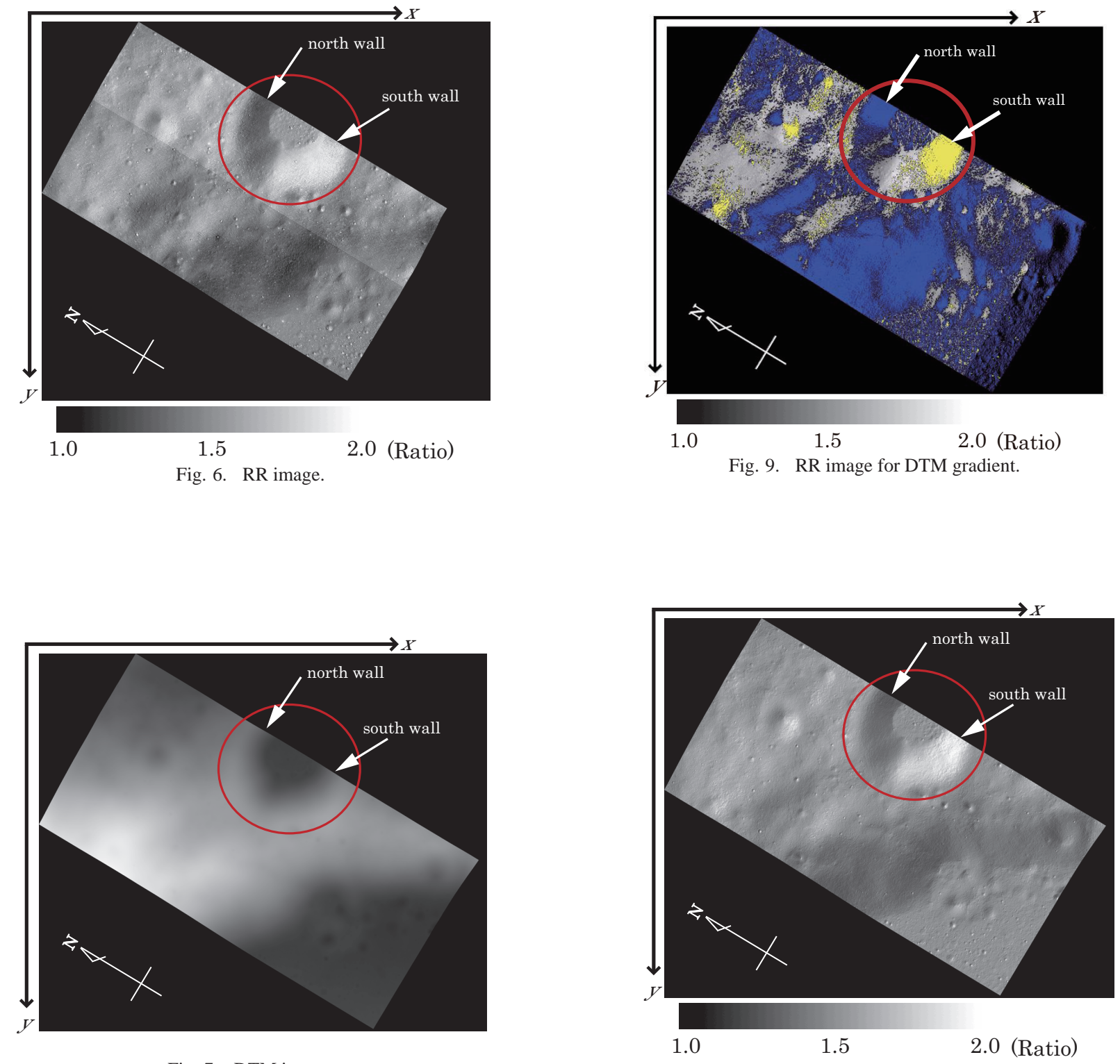

Fig. 7. DTM image

Fig. 10. Mean RR image.
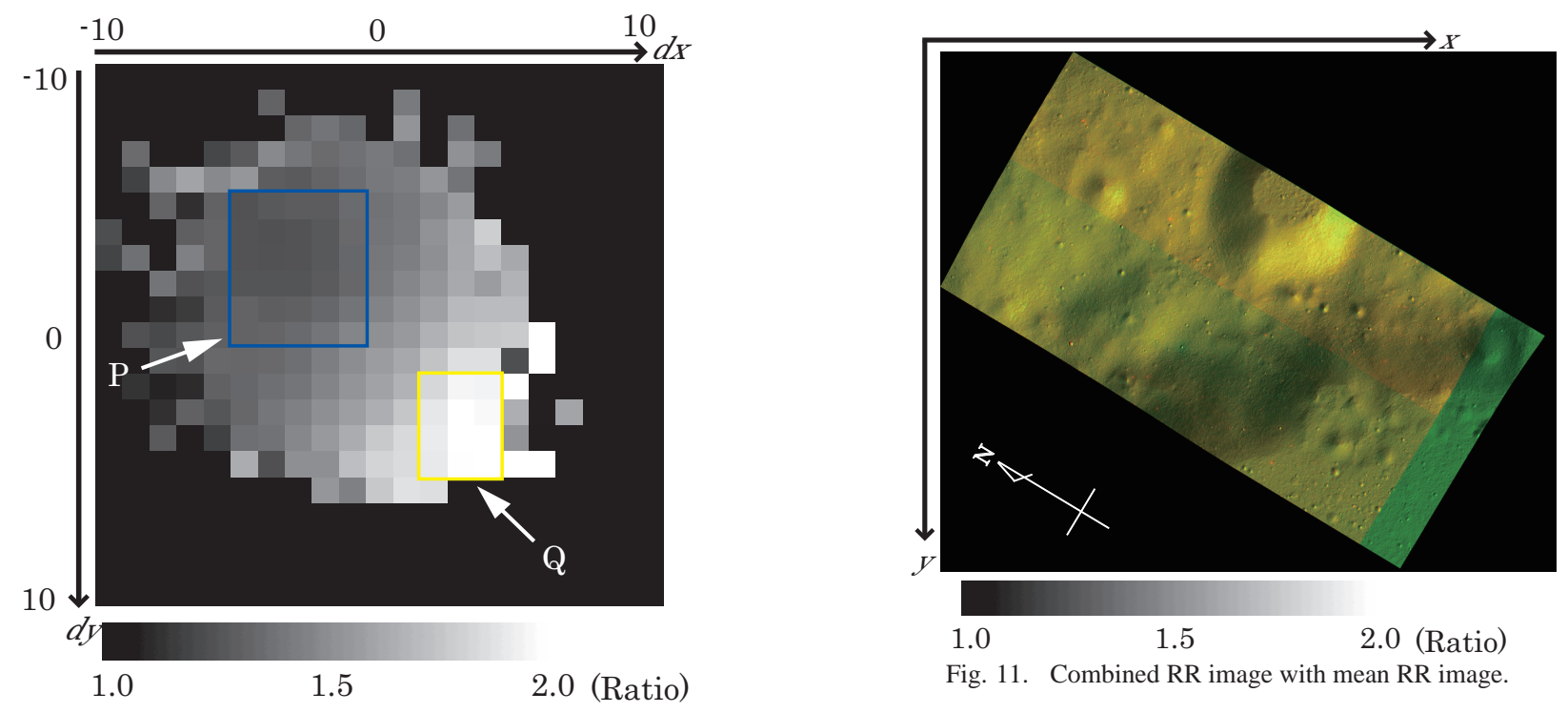

Fig. 11. Combined RR image with mean RR image.

Fig. 8. Mean RR against DTM gradient. 


\section{Conclusion}

It is indicated that the tie point for orthorectification should be set at the midpoint between two line of sight vectors for TC images. The two ortho images from TC1 and TC2 are registered well. The relationship between RR and slope angle is observed. By estimating mean RR of every gradient from RR image and DTM, we make the mean RR for gradient of DTM, in which effects of roughness are reduced. Comparing the RR image with the mean RR image enable to investigate effects of roughness for simple Lambertian reflectance. Finally, we indicated that the lunar surface deviates from Lambert's law. We need researches considering other reflectance models, solar angle, position of orbiter, and so on. Especially, using high level data product of reflectance will lead to quantitative result.

\section{Acknowledgement}

The authors are thankful to Institute of Space and Astronautical Science (ISAS) and LISM members for providing the TC images. I also wish to thank collaborators for their comments and insights.

\section{References}

1) Haruyama, J., Otake, H., Ohtake, M., Shiraishi, A., Hirata, N., Matsunaga, T.: LISM (Lunar Imager/SpectroMeter) Mission for SELENE Project, 31st Lunar Planet. Sci. Conf., abst. no. 1317, 2000.

2) Maejima, H., Sasaki, S., Takizawa, Y.: Development of Selenological and Engineering Explorer (SELENE), Space Resources Roundtable VII, Proc. LEAG Conf. Lunar Expl., 62, No. 1287, 2005.

3) Kato, M., Takizawa, Y., Sasaki, S., Selene Project Team.: The Japanese lunar orbiting satellites mission: present status and science goals, 37th Lunar Planet. Sci. Conf., abst. no. 1233, 2006.
4) Haruyama, J., Ohtake, M., Hirata, N., Nakamura, R., Matsunaga, T. : Expected performance of lunar imager/spectrometer on SELENE, 34th Lunar Planet. Sci. Conf., abst. no. 1565, 2003 a.

5) Haruyama, J., Ohtake, M., Matsunaga, T., Hirata, N.: LISM Working Group. Flight model performance of SELENE terrain camera, Proc. 23th ISTS., pp. 1992-1996, 2003b.

6) Haruyama, J., Ohtake, M., Hirata, N., Nakamura, R., Matsunaga, T. : Flight model performance of SELENE Terrain Camera (II), Proc. of the 25th ISTS., pp. 857-862, 2005.

7) Haruyama, J., Ohtake, M., Matsunaga, T., Morota, T., Yoshizawa, A. : LISM Working Group. Planned digital terrain model products from SELENE terrain camera data, in: 37th Lunar Planet. Sci. Conf., abst. no. 1132,2006 a.

8) Haruyama, J., Ohtake, M., Matsunaga, T.: Global high-resolution stereo mapping of the Moon with the SELENE Terrain Camera, Advances in Geosciences, Ip, W.-H., Bhardwaj, A. (Eds.), Planetary Science, vol. 3. World Scientific Publishing, 2006b, pp. 101-108.

9) Haruyama, J., Matsunaga, T., Morota, T., Honda, C., Torii, M., Yokota, Y., Kawasaki, H., Ohtake, M. : LISM Working Group. Prelaunch operation planning of lunar imager/spectrometer (LISM) on SELENE, in: 38th Lunar Planet. Sci. Conf., abst. no. 1136, 2007.

10) Wilhelms, D.E.: The Geologic history of the Moon, U.S. Geol. Surv. Prof. Pap. 1348, 1987, pp. 302.

11) Haruyama, J., Ohtake, M., Matsunaga, T., Morita, T., Yokota, Y., Honda, C., Hirata, N., Demura, H., Iwasaki, A., Nakamura, R., Kodama, S., LISM Working Group.: Planned radiometrically calibrated and geometrically corrected products of lunar highresolution Terrain Camera on SELENE, J. Adv. Space Res., (2007), doi:10.1016/j.asr.2007.04.062.

12) Mushkin, A. and Gillespie, A.R., Estimating sub-pixcel surface roughness using remotely sensed stereoscopic data, Remote Sensing of Environment, 99 (2005), pp. 75-83.

13) Adams, J. B., Smith, M. O., Gillespie, A. R., Simple models for complex natural surfaces: A strategy for the hyperspectral era of remote sensing, Proc. IEEE International Geosciences Remote Sensing Symposium, vol. I, pp. 16-21, 1989.

14) Hapke, B., Theory of Reflectance and Emmittance Spectroscopy, Topics in remote sensing Vol. 3, New York, Cambridge University Press, 1992. 